\title{
Sodium-Coupled Neutral Amino Acid Transporter 9
}

National Cancer Institute

\section{Source}

National Cancer Institute. Sodium-Coupled Neutral Amino Acid Transporter 9. NCI

Thesaurus. Code C142762.

Sodium-coupled neutral amino acid transporter 9 (561 aa, $\sim 64 \mathrm{kDa}$ ) is encoded by the human SLC38A9 gene. This protein is involved in amino acid sensing, amino acid transport and activation of the mammalian target of rapamycin complex 1 (mT ORC1). 